\title{
Invariant of a Pair of Non-coplanar Conics in Space: Definition, Geometric interpretation and Computation
}

\author{
Long QUAN
}

\author{
LIFIA - CNRS - INRIA, \\ 46, avenue Felix Viallet, \\ 38031 Grenoble, France \\ Email: Long.Quan@imag.fr
}

\begin{abstract}
The joint invariants of a pair of coplanar conics has been widely used in recent vision literature. In this paper, the algebraic invariant of a pair of non-coplanar conics in space is concerned.

The algebraic invariant of a pair of non-coplanar conics is first derived from the invariant algebra of a pair of quaternary quadratic forms by using the dual representation of space conics. Then, this algebraic invariant is geometrically interpreted in terms of cross-ratios. Finally, an analytical procedure for projective reconstruction of a space conic from two uncalibrated images is developed and the correspondence conditions of the conics between two views are also explicited.

Experimentations for the discriminality of the correspondence conditions and the accuracy and stability of the projective reconstruction and of the computation of the invariant are conducted both for simulated and real images.
\end{abstract}

Key words: Invariant, Geometry, Object recognition. 


\section{Introduction}

The study of invariants has recently provoked much interest in the computer vision community, since it is crucial for the development of efficient recognition systems for model based vision. A great deal of work on recognition and shape description using invariants has already been reported, for instance cf. the collection book [26]. Most of the invariants [10, 35, 36, 3, 29, 25] are derived for planar objects using geometric entities such as points, lines and conics from one single image. One of the most used planar invariants is the joint invariants of a pair of planar conics [10]. Some of the algebraic and geometric properties of these invariants are further clarified in [24, 29].

Following the important results of Faugeras [6] and Hartley et al. [15] concerning the projective reconstruction of point sets from the epipolar geometry of the two uncalibrated images, the invariants for the configurations of points and lines in space from two uncalibrated images have been investigated in $[1,14,12,13]$. The invariants from three or more uncalibrated images have also been studied in [28] and [23].

In this paper, we propose to study the invariants of a pair of non-coplanar conics in space from two uncalibrated images. The key idea for computing invariants from two images is the use of the epipolar geometry of the two uncalibrated images. This assumption is still maintained in this paper.

The geometric invariant of a pair of non-coplanar conics has first been mentioned in [13] as illustrated in Figure 1. This geometric invariant is very simply, but unfortunately is not directly computable. In this paper, an algebraic invariant of a pair of non-coplanar conics in space will be defined from the algebra of invariants of quaternary quadratic forms, then its relationship with the geometric invariant will also be established, i.e. the algebraic invariant is geometrically interpreted in terms of cross-ratios which define the geometric invariant. This study is inspired by the last century's mathematical developement on invariants of the quadratic forms $[5,4,33,34,16]$. As the algebraic structure of absolute invariants is much more complicated than that of relative invariants, the mathematicians have mainly been concerned with the relative invariants and have paid little attention for the absolute invariants which are the most useful for computer vision.

After the definition of the algebraic invariant, it remains to compute it effectively from two uncalibrated images. At this stage, the key operation is the projective reconstruction of the space conic from two uncalibrated images. Once the projective reconstruction is done, the invariant can be computed in a straightforward way. A very simple analytical method for projective reconstruction of conics in space will be developed in this paper. The reconstruction procedure is essentially linear in that the two solutions of reconstruction are solved together with only linear computation. Only the extraction of the two different solutions needs to solve a quadratic equation. It is also clarified that the solutions to conic reconstruction are generally ambiguous up to two solutions and is unique only for non transparent objects.

The contributions of this paper are mainly three-fold:

- Definition of an algebraic invariant for a pair of non-coplanar conics in space;

- Geometric interpretation of the algebraic invariant in terms of cross-ratios;

- Algorithm for computing this invariant from two uncalibrated images of the pair of conics.

The remaining sections are organized as follows. In Section 2, the algebraic invariant is derived from the associated quadratic forms using the dual representation of space conics. Then in Section 3 , the relationship between the algebraic invariant and the known geometric invariant is established. In Section 4, the projective reconstruction problem is first formulated in Section 4.2. Section 4.3 gives the correspondence conditions. Next, Section 4.4 gives the analytical method which allows to extract two 
solutions in closed form. Experimental results are presented in Section 5. Finally, some concluding comments are given in Section 6.

Most of algebraic projective geometry terms and results used in the paper can be found in [33]. For a more gentle introduction of invariants for computer vision, one can refer to [26].

\section{Invariants of a pair of non-coplanar conics in space}

\subsection{Number of invariants}

Given a geometric configuration, the number of invariants is roughly speaking the difference of the dimension of the configuration group and the dimension of the transformation group [12, 25] (if the dimension of the isotropy group of the configuration is null). For a pair of non-coplanar conics in $\mathcal{P}^{3}$, there is $1=2 \times(3+5)-(16-1)$ absolute invariant under the action of the general linear group $G L(3)$ in $\mathcal{P}^{3}$. Since each space conic has $8=3+5$ degrees of freedom ( 5 for a conic in a given plane and 3 for the plane in which the conic lies), the dimension of a pair of space conics is $2 \times 8=16$. The transformation group $G L(3)$ is represented by a $4 \times 4$ matrix up to a scaling factor, its dimension is $4 \times 4-1=15$.

\subsection{Geometric invariant}

The unique invariant can be geometrically constructed as illustrated in Figure 1. This has been mentioned in [12]. If the 4 intersection points of the conics with the common line of the conic planes are respectively $P_{1}, P_{2}, P_{3}$ and $P_{4}$ whose projective parameters are $\theta_{1}, \theta_{2}, \psi_{1}$ and $\psi_{2}$. The cross-ratio

$$
\rho=\left(P_{1}, P_{2} ; P_{3}, P_{4}\right)=\left(\theta_{1}, \theta_{2} ; \psi_{1}, \psi_{2}\right)=\frac{\left(\theta_{1}-\psi_{1}\right) /\left(\theta_{2}-\psi_{1}\right)}{\left(\theta_{1}-\psi_{2}\right) /\left(\theta_{2}-\psi_{2}\right)}
$$

is the geometric invariant.

As in all of algebraic geometry, we need to count these 4 intersection points properly, i.e. each of them might be multiple, complex or at infinity.

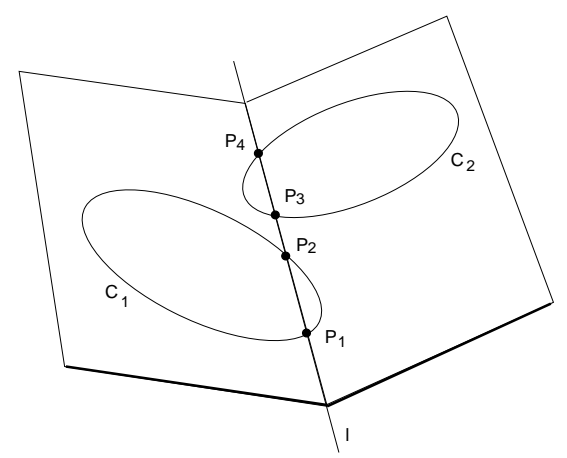

Figure 1: Geometric invariant of a pair of non-coplanar conics: the cross-ratio of the 4 intersection points of the conics with the common line of the conic planes.

\subsection{Space conic as a quadric envelope}

The principle of duality is a fundamental concept in projective geometry. In $\mathcal{P}^{3}$, points and planes are dual to each other. The space dual of a plane curve is a cone. The conic is a quadric envelope of rank 3 which is called a disk quadric [33] 
The plane equation (in plane coordinates instead of usual point coordinates) of the conic, represented as the complete intersection of a proper cone $x^{T} Q x=0$ where $Q$ is a symmetric $4 \times 4$ matrix and a plane $\pi=p^{T} x=0$ in point coordinates, is

$$
\left|\begin{array}{lll}
0 & 0 & u^{T} \\
0 & 0 & p^{T} \\
u & p & Q
\end{array}\right|=0
$$

where $u=\left(u_{1}, u_{2}, u_{3}, u_{4}\right)^{T}$ represents a variable plane.

\subsection{Review of invariants of quaternary quadratic forms}

Since a space conic is a quadric envelope, we first review invariants of a pair of quaternary quadratic forms following Semple and Kneebone [33], Todd [34] and Johnson [16]. Given two quaternary quadratic forms, $\mathcal{Q}_{1} \equiv x^{T} A x$ and $\mathcal{Q}_{2} \equiv x^{T} B x$ in $x$ where $A$ and $B$ are both symmetric $4 \times 4$ matrices, consider the one dimensional family of quadratic forms, i.e. linear combination of $\mathcal{Q}_{1}$ and $\mathcal{Q}_{2}, \mathcal{Q}(\lambda, \mu) \equiv \lambda \mathcal{Q}_{1}+\mu \mathcal{Q}_{2}$. The vanishing of the determinant of $\mathcal{Q}(\lambda, \mu)$ is given by

$$
|\lambda A+\mu B|=0
$$

that is,

$$
I_{1} \lambda^{4}+I_{2} \lambda^{3} \mu+I_{3} \lambda^{2} \mu^{2}+I_{4} \lambda \mu^{3}+I_{5} \mu^{4}=0
$$

where

$$
\begin{aligned}
& I_{1}=|A|, \\
& I_{2}=\sum_{i, j=1}^{4} b_{i j} A_{i j}, \\
& I_{3}=\left|b_{1} b_{2} a_{3} a_{4}\right|+\left|b_{1} a_{2} b_{3} a_{4}\right|+\left|b_{1} a_{2} a_{3} b_{4}\right|+\left|a_{1} b_{2} b_{3} a_{4}\right|+\left|a_{1} b_{2} a_{3} b_{4}\right|+\left|a_{1} a_{2} b_{3} b_{4}\right|, \\
& I_{4}=\sum_{i, j=1}^{4} a_{i j} B_{i j}, \\
& I_{5}=|B|,
\end{aligned}
$$

$a_{i j}$ and $b_{i j}$ are entries of $A$ and $B, A_{i j}$ and $B_{i j}$ cofactors of $a_{i j}$ and $b_{i j}$ in $A$ and $B . A=\left(a_{1} a_{2} a_{3} a_{4}\right)$ and $B=\left(b_{1} b_{2} b_{3} b_{4}\right)$ are the column partition of $A$ and $B$.

When $x$ transforms into $x^{\prime}=T x$ by any non singular space collineation $T, I_{j}$ are transformed into $I_{j}^{\prime}=|T|^{2} I_{j} . I_{j}(j=1, \ldots, 5)$ are relative invariants of weight 2 of the two quaternary quadratic forms $\mathcal{Q}_{1}$ and $\mathcal{Q}_{2}$.

\subsection{Algebraic invariant of a pair of space conics}

Let $C_{1}$ and $C_{2}$ be two space conics, represented by their dual quadric envelopes $\mathcal{C}_{1} \equiv u^{T} A u=0$ and $\mathcal{C}_{2} \equiv u^{T} B u=0$. Consider the invariant algebra of quadratic forms $\mathcal{C}_{1}$ and $\mathcal{C}_{2}$ in $u$. As $\mathcal{C}_{1}=0$ and $\mathcal{C}_{2}=0$ represent conics, so their associated quadric envelopes are of rank 3 , therefore

$$
I_{1}=I_{5}=|A|=|B|=0 .
$$

We are left with $I_{2}, I_{4}$ and $I_{5}$ non vanishing. Two absolute invariants $I_{2}: I_{3}: I_{4}$ can be defined for their associated quadratic forms, however they are not yet the invariants of the conics, since each conic $\mathcal{C}_{i}=0$ is associated with a family of quadratic forms $\lambda_{i} \mathcal{C}_{i}$ for any scalar $\lambda_{i} \neq 0$.

To obtain the absolute invariants of two conics, the power degrees of $\lambda$ and $\mu$ in the family of quadratic forms should also taken into account. When this is done, 


$$
I=\frac{I_{3}^{2}}{I_{2} I_{4}}
$$

Note that the development of this part is formally similar to our previous work on invariants of planar conics.

\section{Geometric interpretation of the algebraic invariant}

As we mentioned earlier, the geometric invariant $\rho$ of two non-planar conics is easily defined as the cross ratio of two pairs of points, each pair of which is the intersection of a conic with the common line of the two conic planes. In this section, we want to interpret geometrically the algebraic invariant $I$ in terms of this geometric invariant $\rho$. The basic idea is to choose particular coordinate representations for quadrics in order to bring them into the simplest forms. The choice is inspired by Semple and Kneebone [33] and Johnson [16].

Without loss of generality, the planes of the two space conics may be taken (up to a space collineation) respectively as $x_{4}=0$ and $x_{1}=0$ in point coordinates, or $(0,0,0,1)^{T}$ and $(1,0,0,0)^{T}$ in plane coordinates.

The plane equations of the conics reduce to

$$
u^{T} A u=u^{T}\left(\begin{array}{cc}
\tilde{A} & 0 \\
0 & 0
\end{array}\right) u=\left(u_{1}, u_{2}, u_{3}\right) \tilde{A}\left(\begin{array}{l}
u_{1} \\
u_{2} \\
u_{3}
\end{array}\right)=0
$$

and

$$
u^{T} B u=u^{T}\left(\begin{array}{cc}
0 & 0 \\
0 & \tilde{B}
\end{array}\right) u=\left(u_{2}, u_{3}, u_{4}\right) \tilde{B}\left(\begin{array}{c}
u_{2} \\
u_{3} \\
u_{4}
\end{array}\right)=0
$$

where $A$ and $B$ are assumed to be symmetric.

The non vanishing relative invariants can be explicited as follows.

$$
\begin{aligned}
& I_{2}=b_{44}|\tilde{A}| \\
& I_{3}=\tilde{A}_{22} \tilde{B}_{33}+\tilde{A}_{33} \tilde{B}_{22}-2 \tilde{A}_{23} \tilde{B}_{23} \\
& I_{4}=a_{11}|\tilde{B}|
\end{aligned}
$$

where $\tilde{A}_{i j}$ and $\tilde{B}_{i j}$ are the cofactors of $a_{i j}$ and $b_{i j}$ in $\tilde{A}$ and $\tilde{B}$.

Now, we require to find two point equations which represent surfaces whose complete intersection is the conic. A convenient pair of surface is the plane of the conic and the cone which joins it to a vertex of the tetrahedron of reference, say the point $(0,0,0,1)^{T}$. The point equation of the cone is

$$
x^{T}\left(\begin{array}{cc}
\tilde{A}^{*} & 0 \\
0 & 0
\end{array}\right) x=\left(x_{1}, x_{2}, x_{3}\right) \tilde{A}^{*}\left(\begin{array}{l}
x_{1} \\
x_{2} \\
x_{3}
\end{array}\right)=0,
$$

where $\tilde{A}^{*}$ is the adjoint matrix of $\tilde{A}$. 
Similarly, taking $(1,0,0,0)^{T}$ as the vertex of the cone associated to the second conic, the point equation of the cone is

$$
x^{T}\left(\begin{array}{cc}
0 & 0 \\
0 & \tilde{B}^{*}
\end{array}\right) x=\left(x_{2}, x_{3}, x_{4}\right) \tilde{B}^{*}\left(\begin{array}{c}
x_{2} \\
x_{3} \\
x_{4}
\end{array}\right)=0,
$$

where $\tilde{B}^{*}$ is the adjoint matrix of $\tilde{B}$.

The common line of the two conic planes is

$$
\left\{\begin{array}{l}
x_{1}=0 \\
x_{4}=0
\end{array}\right.
$$

Therefore the intersection points of the space conics with the common line are the roots of the following binary quadratic equations obtained by respectively setting $x_{1}=0$ and $x_{4}=0$ in the two cones:

$$
\begin{gathered}
\mathcal{B}_{1} \equiv\left(x_{2}, x_{3}\right)\left(\begin{array}{cc}
\tilde{A}_{22} & \tilde{A}_{23} \\
\tilde{A}_{23} & \tilde{A}_{33}
\end{array}\right)\left(\begin{array}{c}
x_{2} \\
x_{3}
\end{array}\right)=\left(x_{2}, x_{3}\right) \bar{A}\left(\begin{array}{c}
x_{2} \\
x_{3}
\end{array}\right)=0, \\
\mathcal{B}_{2} \equiv\left(x_{2}, x_{3}\right)\left(\begin{array}{cc}
\tilde{B}_{22} & \tilde{B}_{23} \\
\tilde{B}_{23} & \tilde{B}_{33}
\end{array}\right)\left(\begin{array}{c}
x_{2} \\
x_{3}
\end{array}\right)=\left(x_{2}, x_{3}\right) \bar{B}\left(\begin{array}{c}
x_{2} \\
x_{3}
\end{array}\right)=0 .
\end{gathered}
$$

At the same time, we can consider the invariant algebra of the two binary quadratic forms $\mathcal{B}_{1}$ and $\mathcal{B}_{2}$, according to $[5,4]$, there are three relative invariants of weight 2 which are

$$
i_{1}=|\bar{A}|, \quad i_{2}=\tilde{A}_{22} \tilde{B}_{33}+\tilde{A}_{33} \tilde{B}_{22}-2 \tilde{A}_{23} \tilde{B}_{23}, \quad \text { and } \quad i_{3}=|\bar{B}| .
$$

For the invariants of two quadratic equations $\mathcal{B}_{1}=0$ and $\mathcal{B}_{2}=0$, the unique absolute invariant is

$$
\mathcal{I}=\frac{i_{2}^{2}}{i_{1} i_{3}}
$$

It is easily verified that $i_{2}=I_{2}, i_{1}=a_{11}|\tilde{A}|$ and $i_{3}=b_{44}|\tilde{B}|$. Thus,

$$
I=\frac{I_{3}^{2}}{I_{2} I_{4}}=\frac{i_{2}^{2}}{i_{1} i_{3}}=\mathcal{I}
$$

The geometric interpretation of the algebraic invariant $I$ is now equivalent to establish the relationship between the algebraic invariant $\mathcal{I}$ of two binary quadratic equations and two pairs of the roots of the quadratic equations. This can be easily achieved as follows.

On the one hand, it is known (cf. [5]) that two binary quadratic forms can be simultaneously reduced to canonical binary forms by a suitable non singular homography:

$$
\left\{\begin{array}{l}
a_{1}^{\prime} x_{2}^{\prime 2}+c_{1}^{\prime} x_{3}^{\prime 2}=0 \\
a_{2}^{\prime} x_{2}^{\prime 2}+c_{2}^{\prime} x_{3}^{\prime 2}=0
\end{array}\right.
$$

thus

$$
I=\mathcal{I}=\mathcal{I}^{\prime}=\frac{\left(a_{1}^{\prime} c_{2}^{\prime}+a_{2}^{\prime} c_{1}^{\prime}\right)^{2}}{a_{1}^{\prime} c_{1}^{\prime} a_{2}^{\prime} c_{2}^{\prime}}
$$


On the other hand, let $\left(\theta_{1}, \theta_{2}\right)$ and $\left(\psi_{1}, \psi_{2}\right)$ be the roots of the binary quadratic equations, given by

$$
\theta^{2}=\left(x_{2}^{\prime}: x_{3}^{\prime}\right)^{2}=-c_{1}^{\prime}: a_{1}^{\prime} \text { and } \psi^{2}=\left(x_{2}^{\prime}: x_{3}^{\prime}\right)^{2}=-c_{2}^{\prime}: a_{2}^{\prime}
$$

The geometric invariant is the cross ratio $\rho=\left(\theta_{1}, \theta_{2} ; \psi_{1}, \psi_{2}\right)$. There exists the following relationship between $\rho$ and $I$

$$
I=4\left(\frac{\rho+1}{\rho-1}\right)^{2}
$$

Obviously, $\rho=-1$ turns out to be the geometric interpretation of the vanishing of $I_{3}$.

\section{Projective reconstruction of the conics in space}

The algebraic invariant of a pair of non-coplanar conics is defined in space, that is in $\mathcal{P}^{3}$. It remains to reconstruct the space conic from image conics which are in $\mathcal{P}^{2}$. This section will give an algorithm for projective reconstruction of a space conic from its two uncalibrated images.

At first, some basic concepts related to camera geometry are briefly presented in Section 4.1. For more details, one can refer to [7].

\subsection{Preliminaries}

Projection matrix of a camera If we assume a perspective projection for camera model, then the object space $\mathcal{R}^{3}$ may be considered as embedded in $\mathcal{P}^{3}$ (projective space of dimension 3) and the image space $\mathcal{R}^{2}$ embedded in $\mathcal{P}^{2}$. The camera performs the projection between $\mathcal{P}^{3}$ and $\mathcal{P}^{2}$, and this projection is represented by a $3 \times 4$ matrix $P$ of rank 3 whose kernel is the projection center, $P$ is called the projection matrix of the camera. The relation between an image point in its homogeneous coordinates $\tilde{x}=(u, v, w)^{T}$ in $\mathcal{P}^{2}$ and a space point in its homogeneous coordinates as well $x=\left(x_{1}, x_{2}, x_{3}, x_{4}\right)^{T}$ in $\mathcal{P}^{3}$ are linearly related by $\lambda \tilde{x}^{T}=P x$, or

$$
\lambda(u, v, w)^{T}=P\left(x_{1}, x_{2}, x_{3}, x_{4}\right)^{T} .
$$

Uncalibrated images When $P$ is totally unknown for a given image, we say that this image is uncalibrated. For a pair of images, $P$ may be unkown for each image, however the epipolar geometry of a pair of uncalibrated images can be estimated from only the point correspondences of the two images. It is therefore more appropriate to call the pair of uncalibrated images with known epipolar geometry as the weakly, as suggested in [31] or the projectively calibrated pair of images.

In this paper, we assume that the epipolar geometry is always given for the pair of uncalibrated images. In fact, the epipolar geometry is the key for the projective reconstruction from two uncalibrated images as demonstrated in $[6,15]$.

The epipolar geometry can be nicely coded by a $3 \times 3$ rank 2 matrix $F$, called fundamental matrix $[19,8]$ or essential matrix [15]. According to Hartley [15], one possible choice of projection matrices for two cameras consistent with a decomposition of $F=M[t]_{x}$ is given by

$$
P=\left(\begin{array}{ll}
I_{3} & 0_{3}
\end{array}\right) \text { and } P^{\prime}=\left(M^{*}-M^{*} t\right)
$$


where $M$ is a non singular $3 \times 3$ matrix, $t=\left(t_{1}, t_{2}, t_{3}\right)^{T} . M^{*}$ is the adjoint matrix of $M$, and $[t]_{x}$ is the antisymmetric $3 \times 3$ matrix associated to the vector $t$. For more details, see [15].

The reconstruction in $\mathcal{P}^{3}$ is therefore defined up to the projective transformation of the placement of the first camera.

\subsection{Formulation of projective reconstruction}

In the following, without explicit mention, the pair of projection matrices $P$ and $P^{\prime}$ are defined up to projective transformations. We are therefore working in projective spaces which allow to reveal more intrinsic properties of the conic reconstruction. The Euclidean case can be proceeded in exactly the same way by taking the suitable projection matrices, this will be discussed in Section 4.5.

Given a corresponding pair of conics in two distinct images,

$$
\mathcal{C} \equiv \tilde{x}^{T} C \tilde{x}=0 \leftrightarrow \mathcal{C}^{\prime} \equiv \tilde{x}^{T} C^{\prime} \tilde{x}^{\prime}=0
$$

we require to find a conic in space which has been projected respectively into $\mathcal{C}$ and $\mathcal{C}^{\prime}$. A conic in space is generally represented as the complete intersection of a quadric surface and a plane. The reconstruction is therefore equivalent to find the plane in which the conic lies, as we can take any one of the two cones associated to two conics in images as the quadric surface.

The cone equation associated to a given conic and a given camera is obtained as follows.

Given a projection matrix $P$ of a camera, the equation of the cone which joins the conic $\tilde{x}^{T} C \tilde{x}=0$ in the image plane to the projection center of the camera is $x^{T} Q x=x^{T} P^{T} C P x=0$.

This can be easily proved by substituting $\lambda \tilde{x}^{T}=P x$ into the conic equation $\tilde{x}^{T} C \tilde{x}=0 . x^{T} Q x=0$ is effectively a proper cone, $\operatorname{for}^{1} \operatorname{rank}(Q)=\operatorname{rank}(P)=\operatorname{rank}(C)=3$ and $\operatorname{Ker}(Q)=\operatorname{Ker}(P)$ which is meant that the vertex of the cone is the projection center of the camera.

The cones corresponding to the pair of conics are respectively

$$
\mathcal{Q} \equiv x^{T} A x=x^{T} P^{T} C P x=0 \quad \text { and } \quad \mathcal{Q}^{\prime} \equiv x^{T} B x=x^{T} P^{\prime T} C^{\prime} P^{\prime} x=0
$$

in $\mathcal{P}^{3}$.

Consider the pencil of quadric surfaces $\mathcal{Q}+\lambda \mathcal{Q}^{\prime}=0$, for every value of $\lambda$ the equation $\mathcal{Q}+\lambda \mathcal{Q}^{\prime}=0$ represents a quadric surface which passes through all the common points of $\mathcal{Q}$ and $\mathcal{Q}^{\prime}$. The points common to all quadric surfaces of the pencil are simply the points which make up the curve of intersection of $\mathcal{Q}$ and $\mathcal{Q}^{\prime}$, and this curve is the base curve of the pencil. The base curve of two quadric surfaces is generally a quartic curve. In our context, the reconstruction constraints impose that the corresponding cones intersect in a conic in space. As this conic in space should be part of the base curve, thus the base curve of the pencil should break up and one of the components is a conic in space. Even more, if one of the components of the base curve is a conic, the residual component should also be a conic. As a pair of planes can be considered as a degenerate quadric surface of rank 2, according to the results of projective geometry (cf. [33]) on pencils of quadric surfaces, the degenerate quadric surface composed of the pair of planes belongs to the pencil of quadric surfaces in consideration. We are therefore led to examine a special pencil of quadric surfaces which contains a degenerated member of rank 2. Based on this, we can reformulate the problem of conic reconstruction as follows:

\footnotetext{
${ }^{1}$ For a quadric surface $x^{T} A x=0$, the projective classification of quadric surfaces (cf. [33]) based on the rank of $A$ is given as: a proper quadric surface has full rank 4, a proper quadric cone rank 3, a pair of distinct planes rank 2 and a repeated plane rank 1 .
} 
The reconstruction of a conic in space from two images is equivalent to find a $\lambda$ such that the $\lambda$-matrix $C(\lambda)=A+\lambda B$ has rank 2. $x^{T} A x=0$ and $x^{T} B x=0$ are the proper cones corresponding to the two images of the conic in space.

We can also think that we may have the case where the pair of planes coincident, becoming a repeated plane which is a degenerate quadric surface of rank 1 . If this was possible, the reconstruction would be unique. However, in the following, we show that it is impossible.

If the conics in images are proper, there is no $\lambda$ such that $C(\lambda)=A+\lambda B$ can have rank 1.

This will be proved in the next section after other results will have been introduced.

\subsection{Correspondence conditions}

Unlike points and lines, two images of a conic in space contain sufficient information to impose correspondence conditions. The number of the independent conditions which can be derived is established as follows:

There exist two independent polynomial conditions for a corresponding pair of conics.

To prove it, we need only to count the degrees of freedom of the rank 2 matrix and those of the matrix pencil. A $4 \times 4$ symmetric matrix up to a scaling factor counts for $10-1=9$ degrees of freedom, thus a general matrix pencil counts for $9-1=8$ degrees of freedom. A rank 2 symmetric matrix $C$ of order 4 counts for 6 degrees of freedom, so there remain $2=8-6$ independent conditions.

We will now derive these two polynomial conditions.

Consider the characteristic polynomial of $\lambda$-matrix $C(\lambda)=A+\lambda B$,

$$
|C(\lambda)-\mu I|=\mu^{4}+a_{1}(\lambda) \mu^{3}+a_{2}(\lambda) \mu^{2}+a_{3}(\lambda) \mu+a_{4}(\lambda)=0
$$

$C(\lambda)$ is a real symmetric $4 \times 4$ matrix. For it to have rank 2 , it must have two distinct nonzero eigenvalues and a double zero eigenvalue. The conditions we are looking for are equivalent to have ${ }^{2}$

$$
\left\{\begin{array}{l}
a_{3}(\lambda)=0 \\
a_{4}(\lambda)=0
\end{array}\right.
$$

By definition, $a_{4}(\lambda)$ is the determinant of $C(\lambda)$, therefore, it is the characteristic polynomial of the matrix pencil $A+\lambda B$, i.e.

$$
a_{4}(\lambda)=|C(\lambda)|=|A+\lambda B|=I_{1} \lambda^{4}+I_{2} \lambda^{3}+I_{3} \lambda^{2}+I_{4} \lambda+I_{5}
$$

where the coefficients $I_{j}$ are polynomials in the entries of $A$ and $B$.

Since $A$ and $B$ both have $\operatorname{rank} 3, I_{1}=|A|=0$ and $I_{5}=|B|=0$. The characteristic polynomial of the pencil is factorized as

$$
a_{4}(\lambda)=\lambda\left(I_{2} \lambda^{2}+I_{3} \lambda+I_{4}\right)=0
$$

There are generally four singular matrices of the pencil, each corresponds to one of the four generalized eigenvalues of the pencil, the roots of $a_{4}(\lambda)=0$. Two generalized eigenvalues of the pencil

\footnotetext{
${ }^{2}$ To be complete, we should also have $a_{2} \neq 0$ and $a_{1}^{2}-4 a_{2} \neq 0$ to guarantee two distinct nonzero eigenvalues.
} 
are easily read out as $\lambda=0$ and $\lambda=\infty$ which corresponds respectively to $A$ and $B$. The two others are the solutions of the quadratic equation

$$
I_{2} \lambda^{2}+I_{3} \lambda+I_{4}=0
$$

Before going ahead, let us recall the following:

Lemma 1 Given symmetric matrices $A$ and $B$ of order $n$. If $\lambda_{i}$ is a generalized eigenvalue of $A+\lambda B$ of multiplicity $k_{i}$, then $k_{i}$ is at least $n-m$ for $C\left(\lambda_{i}\right)=A+\lambda_{i} B$ to have rank $m$.

Proof. This is a direct consequence of the fact that the dimension of nullspace of $A+\lambda_{i} B$ can not exceed $k_{i}$.

In order to have a rank 2 matrix in the pencil, we should at least have a generalized eigenvalue of multiplicity 2 , hence the above quadratic equation (6) must have two equal roots. The condition for this is

$$
\Delta \equiv I_{3}^{2}-4 I_{2} I_{4}=0
$$

that is the first condition for correspondence.

It is now straightforward that we can not have a rank 1 matrix in this matrix pencil. If so, we should at least have a triple generalized eigenvalue which is obviously impossible for we have already two distinct ones 0 and $\infty$. This proves the Proposition 4.2 .

After $a_{4}(\lambda)=0$ of $(5)$ is examined, we can now examine $a_{3}(\lambda)=0 . a_{3}(\lambda)$ is a cubic polynomial in $\lambda$, it can be written as

$$
a_{3}(\lambda)=J_{1} \lambda^{3}+J_{2} \lambda^{2}+J_{3} \lambda+J_{4}
$$

where $J_{i}$ are polynomials in the entries of $A$ and $B$.

The second condition is derived by computing the resultant of $a_{3}(\lambda)$ and $a_{4}(\lambda)$ with respect to $\lambda$. The explicit form of this resultant, further simplified by using $\Delta=0$, gives the second polynomial condition

$$
\Theta \equiv-J_{1} I_{3}^{3}+2 J_{2} I_{3}^{2} I_{2}-4 J_{3} I_{3} I_{2}^{2}+8 J_{4} I_{2}^{3}=0
$$

The results can then be summarized in

The two polynomial correspondence conditions for a pair of corresponding conics are respectively $\Delta=0$ and $\Theta=0$.

Remark The second correspondence condition is a polynomial of higher degrees than the first one. The experiments conducted in Section 5 will show that the first condition is much more discriminant than the first one. In practice, only the first condition will be used to establish the correspondences of conics between two images. Interestingly, the first condition $\Delta=0$ admits a nice invariant interpretation.

Let us first consider the invariant algebra of the pair of the quadratic forms $x^{T} A x$ and $x^{T} B x$ as in the previous Section 2. They have 5 relative invariants $I_{j}, j=1, \ldots, 5$ of weight 2 of the two quaternary quadratic forms $x^{T} A x$ and $x^{T} B x$. 
Now let take the quaternary quadratic forms of a pair of cones $x^{T} A x=0$ and $x^{T} B x=0$, so $I_{1}=I_{5}=|A|=|B|=0$. We are left with nonzero $I_{2}, I_{4}$ and $I_{5}$. While taking into account the power degrees of $\lambda$ and $\mu$, the unique absolute invariant of a pair of the cones is

$$
I=\frac{I_{3}^{2}}{I_{2} I_{4}}
$$

As $\Delta=I_{3}^{2}-4 I_{2} I_{4}$, therefore

$$
\Delta=0 \Leftrightarrow I=4 . \square
$$

\subsection{Closed-form solutions of reconstruction}

The degenerate quadric surface Since we must have two equal roots for the quadratic equation (6), the double generalized eigenvalue is directly obtained by

$$
\lambda=-\frac{I_{3}}{2 I_{2}}
$$

Then we obtain the matrix $C=A+\lambda B$ of the degenerate quadric surface.

The remaining effort for conic reconstruction requires only to extract the two planes from this rank 2 matrix $C$.

Extraction of the plane pair The extraction of the plane pair from $C$ consists of the eigen analysis of $C$, which can be directly solved as follows.

Going back to the characteristic polynomial of the matrix $C(\lambda)$, it is simplified by the second condition $\Theta=0$ as

$$
\mu^{2}\left(\mu^{2}+a_{1}(\lambda) \mu+a_{2}(\lambda)\right)=0
$$

The remaining two nonzero eigenvalues $\mu_{1}$ and $\mu_{2}$ are the roots of the quadratic equation:

$$
\mu^{2}+a_{1}(\lambda) \mu+a_{2}(\lambda)=0
$$

As $C$ is a real symmetric matrix, there exists a non singular transformation $T$ such that $C$ is diagonalized:

$$
T^{T} C T=\operatorname{diag}\left(\mu_{1}, \mu_{2}, 0,0\right)
$$
i.e.

The quadric surface $x^{T} C x=0$ is therefore transformed by $x=T x^{\prime}$ into $x^{\prime T} \operatorname{diag}\left(\mu_{1}, \mu_{2}, 0,0\right) x^{\prime}=0$,

$$
\mu_{1} x_{1}^{\prime 2}+\mu_{2} x_{2}^{\prime 2}=0
$$

The pair of planes $\pi_{i}^{\prime} \equiv p_{i}^{\prime T} x^{\prime}=0, i=1,2$ in the transformed reference frame is

$$
\left(\sqrt{\mu_{1}}, \pm \sqrt{-\mu_{2}}, 0,0\right)^{T} x^{\prime}=\sqrt{\mu_{1}} x_{1}^{\prime} \pm \sqrt{-\mu_{2}} x_{2}^{\prime}=0 .
$$

It is obvious that to obtain real planes, we must have ${ }^{3}$

\footnotetext{
${ }^{3}$ This inequality condition does not affect the degree of freedom of the matrix pencil, so it does not affect the Proposition 4.3.
} 


$$
a_{2}(\lambda)=\mu_{1} \mu_{2}<0
$$

Let $v_{1}$ and $v_{2}$ be the eigenvectors corresponding to the eigenvalues $\mu_{1}$ and $\mu_{2}$ of $C$. The plane pair $\pi_{i} \equiv p_{i}^{T} x=0, i=1,2$ in the original reference frame are obtained by

$$
\left(T p_{i}^{\prime}\right)^{T} x=\left(\sqrt{\mu_{1}} v_{1} \pm \sqrt{-\mu_{2}} v_{2}\right)^{T} x=0
$$

Then the conic in space is defined as the intersection of one of the two cones with the plane recovered above.

Determination of the unique plane from the plane pair At this stage, the reconstruction of conics in space is ambiguous up to two solutions, defined by any plane of the plane pair. This ambibuity cannot be removed in the pure projective 3 space $\mathcal{P}^{3}$ except that if we further suppose that conics contain no real points at infinity (i.e. only ellipses). In Euclidean case, this ambiguity can be removed for non-transparent objects, this point will be discussed in Section 4.5.

Summary of the computation From a pair of stereo images, after the conic features from two images have been extracted, the correspondence between two images and the reconstruction are solved by the following procedure.

1. Selection of a corresponding pair of conics

(a) For each pair of conics $C_{i}$ and $C_{j}^{\prime}$, form the cones $A=P^{T} C_{i} P$ and $B=P^{\prime T} C_{j}^{\prime} P^{\prime}$, compute $I_{2}, I_{3}$ and $I_{4}$, then $\Delta_{i, j}$.

(b) A potential corresponding pair of conics is selected as the pair that has the smallest absolute value of $\Delta_{i, j}$ among all possible pairs of conics between two images.

(c) If $\left|\Delta_{i, j}\right|<\epsilon$, it is considered as a pair of corresponding pair, else no correspondence for the given $C_{i}$.

2. Projective reconstruction

(a) Take $\lambda=-I_{3} / 2 I_{2}$, compute $C=A+\lambda B$,

(b) Extract the two planes

i. If $a_{2}>0$, solve the quadratic equation (7) for $\mu_{1}$ and $\mu_{2}$, else no real solution possible.

ii. Compute the eigenvectors $v_{1}$ and $v_{2}$ associated to $\mu_{1}$ and $\mu_{2}$ by solving linear equation $\left(C-\mu_{i} I\right) v_{i}=0$,

iii. Plane pair is given as $\left(\sqrt{\mu_{1}} v_{1} \pm \sqrt{-\mu_{2}} v_{2}\right)^{T} x=0$

3. Computation of the invariant

(a) Form the plane equation of each possible space conic according to the formule (1);

(b) Computation of the relative invariants of any pair of space conics expressed in plane equation.

(c) Form the absolute invariant using the formule (2). 


\subsection{Related work on conic reconstruction}

It is also important to note that several authors have remarked the importance of conics as basic image features and developed some procedures for pose estimation, stereo and motion based on conics, for instance $[18,2,20,32,26,37,9,22]$. The conic reconstruction algorithm proposed in this paper is related to but different from those of Ma et. al [21, 20] and Safaee-Rad et. al [32]. They both have been interested in the Euclidean reconstruction of space conic and proposed different solutions to the problem.

Ma et al. in $[21,20]$ developed a different analytical method which reconstructs directly the position and orientation of the conic in space, and proposed a criterion for correspondence verification. The approach was developed and heavily limited to Euclidean framework, hence some properties regarding projective quadrics cannot be revealed in this framework. The matching criterion is more a a posteriori verification procedure, mixed up with the reconstruction procedure.

In [32], Safaee-Rad et al. observed the projective property of a pencil of quadrics, then proposed a procedure to reconstruct the plane on which the conic in space lies. However, the solution proposed by Safaee-Rad et al. needs to solve a high (fourth) degree polynomial, this is due to lack of further investigation of the problem. The important properties related to the special pencil of matrices are not exploited. The independency of derived equations was not analysed, therefore the correspondence conditions could not be explicited. The uniqueness issue of reconstruction has also been discussed in $[32]$.

The projective reconstruction of space conic can be easily extended to Euclidean reconstruction when the stereo system is strongly calibrated. In the Euclidean geometry framework, it is meant that we are given the 5 intrinsic parameters for each camera, coded respectively as the entries of an upper triangular $3 \times 3$ matrix $A$ and $A^{\prime}$ of form $\left(\begin{array}{ccc}\alpha_{u} & s & u_{0} \\ 0 & \alpha_{v} & v_{0} \\ 0 & 0 & 1\end{array}\right)$ and a rigid displacement between two cameras, represented by a rotation matrix $R$ and a translation vector $t$. The following pair of projection matrices can be assigned to the two cameras

$$
P=A\left(I_{3} 0_{3}\right) \quad \text { and } \quad P^{\prime}=A^{\prime}\left(I_{3} 0_{3}\right)\left(\begin{array}{cc}
R & t \\
0_{3}^{T} & 1
\end{array}\right)
$$

where $I_{3}$ is the $3 \times 3$ identity matrix.

The $3 \mathrm{D}$ reconstruction in $\mathcal{R}^{3}$ is therefore defined up to the rigid transformation of the placement of the first camera.

In fact, whether we are given a strongly calibrated stereo system or a weakly calibrated one, it is equivalent to be given a pair of projection matrices $P$ and $P^{\prime}$. The difference resides in that $P$ and $P^{\prime}$ are defined up to a rigid transformation of the placement of the first camera in the case of the strong calibration case and up to a projective transformation in the case of the weak calibration. The resulting reconstruction will be Euclidean or projective according to that the pair of projection matrices is defined up to a Euclidean or projective transformation.

As we have mentioned that in case of Euclidean reconstruction, the ambiguity of double solution can be removed if we suppose that the conic in space is a non transparent object (a wired conic in space is a typical example of transparency). The visibility constraint may be used to get rid of the surplus solution. To be visible for a non transparent plane from two different viewpoints, it is necessary to have the two view points located on the same side of the plane. Look at Figure 2 in which one plane of the reconstructed pair of planes is always between the two camera centers, therefore this plane is not the solution of the problem.

This can be easily checked as follows. The projection centers of cameras, given their projection 


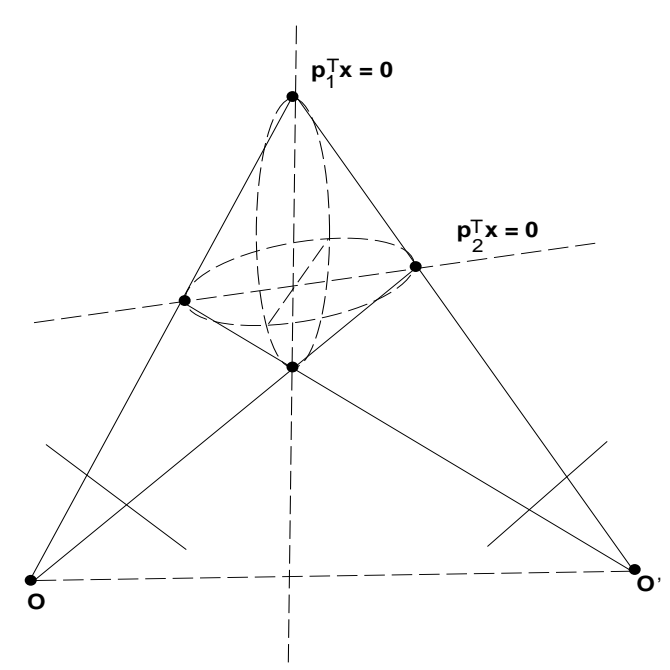

Figure 2: This figure illustrates that one of the pair of planes is always between two camera centers, therefore it is not the solution of the problem if we are considering non transparent objects. One side of the plane $p_{2}^{T} x=0$ is visible for both cameras, however each side of the plane $p_{1}^{T} x=0$ is visible by a different camera.

matrices $P=\left(P_{3 \times 3} p\right)$ and $P^{\prime}=\left(P_{3 \times 3}^{\prime} p^{\prime}\right)$ are

$$
O=\left(\begin{array}{c}
-P_{3 \times 3}^{-1} p \\
1
\end{array}\right) \quad \text { and } \quad O^{\prime}=\left(\begin{array}{c}
-P_{3 \times 3}^{\prime-1} p^{\prime} \\
1
\end{array}\right)
$$

If $\left(O^{T} p_{i}\right)\left(O^{\prime T} p_{i}\right)>0$ then $O$ and $O^{\prime}$ lie both on the same side of the plane $p_{i}^{T} x=0$, else they lie on different sides of the plane. Thus this allows us to remove the reconstruction ambiguity.

It is also important to note that this test can also be extended to the projective case if we further suppose that conics contain no real points at infinity (i.e. only ellipses) because the visibility is still valid for objects containing no points at infinity in the weak calibration case [31].

\section{$5 \quad$ Experimental results}

The theoretical results presented above for corresponding conditions and reconstruction of conics have been implemented. In Section 5.1 and 5.2, the discriminality of correspondence conditions and Euclidean reconstruction of conics are studied both for simulated and real images. In Section 5.3, the experimental results on projective reconstruction and the computation of the invariants are presented.

\subsection{The experiments with simulated images}

\subsubsection{Simulation set-up}

- We use the calibration matrices from a real stereo system. They are given by

$$
\begin{aligned}
P & =\left(\begin{array}{cccc}
1.393757 & -0.244708 & -14.170794 & 368.0 \\
10.624195 & 2.396275 & -0.433595 & 202.0 \\
0.002859 & 0.011811 & -0.003481 & 1.0
\end{array}\right), \\
P^{\prime} & =\left(\begin{array}{cccc}
1.374060 & -0.612998 & -14.189693 & 371.0 \\
10.979978 & -1.621189 & -0.469463 & 207.0 \\
0.007648 & 0.010572 & -0.003449 & 1.0
\end{array}\right)
\end{aligned}
$$


- We are viewing with these two cameras two conics in space described respectively by the intersection of the quadric surface $x^{T} Q_{1} x=0$ and the plane $p_{1}^{T} x=0$ for the first conic in space and the intersection of $x^{T} Q_{2} x=0$ and $p_{2}^{T} x=0$ for the second. The plane $p_{2}^{T} x=0$ is taken as being parallel to the first image plane and goes through the center of $x^{T} Q_{2} x=0$.

$$
\begin{aligned}
Q_{1} & =\left(\begin{array}{cccc}
-0.0013 & 0.4710^{-5} & -0.00023 & 0.0058 \\
0.4710^{-5} & -0.000078 & -0.00034 & 0.0033 \\
-0.00023 & -0.00034 & -0.0014 & 0.011 \\
0.0058 & 0.0033 & 0.011 & -0.038
\end{array}\right) \text { and } p_{1}=(-0.021,-0.16,-0.092,1.0)^{T} \\
Q_{2} & =\left(\begin{array}{cccc}
1.0 & 0.0 & 0.0 & -9.0 \\
0.0 & 1.0 & 0.0 & -2.0 \\
0.0 & 0.0 & 1.0 & -10.0 \\
-9.0 & -2.0 & -10.0 & 85.0
\end{array}\right) \text { and } p_{2}=(-0.196589,-0.812143,0.239359,1.0)^{T}
\end{aligned}
$$

- The conic in space is analytically projected into image planes by the two calibration matrices.

- The projected conics in images are resampled as a list of points. Each location of resampled points is perturbed by varying levels of pixel noise of a uniform distribution.

- Each list of perturbed points is then fitted to a conic of form

$$
a x^{2}+b x y+c y^{2}+d x+e y+f=0
$$

With the normalization $f=1$, least squares fitting can be easily implemented as a linear minimization procedure. Note that using directly this normalization fails for all conics through the origin (as in this case $f=0$ ), this can be easily overcome by shifting the data away from the origin if necessary. In practice, very good results are obtained using this normalization. More sophisticated fitting algorithms are only necessary when a small part of curve data is visible and/or the curve data is very scattered. A more detailed description on conic fitting can be found in $[2,17]$.

\subsubsection{Reconstruction stability w.r.t. pixel errors}

Conic reconstruction with respect to different pixel errors of image points is performed to demonstrate its stability. Table 1 and 2 show the numerical results for the two conics in space. As conic reconstruction is ambiguous, the solution for the plane which is closer to the known space plane of the conic is marked in bold font. Numerically, we can never have a perfect rank 2 matrix $C$. It is well known that numerical rank is nicely characterised in terms of SVD [11]. In the tables of results, we also computed the ratio $\sigma_{3} / \sigma_{2}$ of the second largest $\sigma_{2}$ and the third largest singular values $\sigma_{3}$, which indicates how near $C$ is to a rank 2 matrix. The closer to zero of $\sigma_{3} / \sigma_{2}$, the nearer $C$ is to rank 2 .

We notice that for both space conics, the degradation with the increasing pixel noise is extremely graceful. This is greatly due to that conics are global primitives, small pixel errors are very well corrected by the fitting process.

\subsubsection{Discriminality of correspondence condition}

To demonstrate the discriminality of correspondence conditions, a set of very similar conics is generated by deforming the initial one. The deformation is performed by adding different levels of pixel 


\begin{tabular}{|c|c|c|c|c|c|c|}
\hline Noise & $\overline{\Delta \Delta}$ & $\overline{\bar{\Theta}}$ & $\overline{\sigma_{3} / \sigma_{2}}$ & 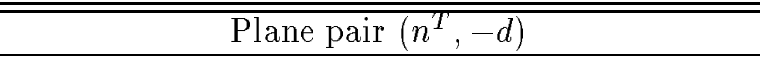 & 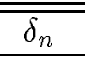 & $\overline{\overline{\delta_{d}}}$ \\
\hline \pm 1.5 & $0.710^{-5}$ & $0.710^{-10}$ & $0.3910^{-2}$ & $\begin{array}{c}\mathbf{( - 0 . 1 1 5 4 8 7 ,}-\mathbf{- 0 . 8 6 1 9 9 1 ,} \mathbf{- 0 . 4 9 3 5 9 3 , ~ 5 . 3 8 1 5 0 )} \\
(-0.970367,0.240090,0.0273005,2.25818)\end{array}$ & 0.17 & 0.002 \\
\hline \pm 2.5 & $-0.310^{-4}$ & $0.1310^{-10}$ & $0.2610^{-2}$ & $\begin{array}{c}\mathbf{- 0 . 1 0 8 0 4 4 ,}, \mathbf{- 0 . 8 6 3 6 8 8 ,}, \mathbf{- 0 . 4 9 2 3 1 0 , 5 . 3 8 4 9 7 9 )} \\
(-0.969659,0.242676,0.0295063,2.23849)\end{array}$ & 0.36 & 0.002 \\
\hline \pm 3.5 & $-0.510^{-4}$ & $0.910^{-10}$ & $0.7210^{-2}$ & $\begin{array}{c}\mathbf{- 0 . 1 3 2 3 0 0 ,}-\mathbf{- 0 . 8 6 3 6 8 4}, \mathbf{- 0 . 4 8 6 3 6 2 , ~ 5 . 3 0 8 0 4 )} \\
(-0.969236,0.244226,0.0305695,2.28233)\end{array}$ & 1.2 & 0.08 \\
\hline \pm 4.5 & $0.110^{-2}$ & $0.510^{-9}$ & 0.024 & 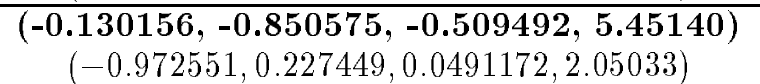 & 1.42 & 0.07 \\
\hline
\end{tabular}

Table 1: Reconstruction results for the first simulated conic in space with different pixel errors for image points before fitting. $\sigma_{2}$ and $\sigma_{3}$ are the second and the third largest singular values of the plane pair matrix. $\delta_{n}$ is the difference of normal direction $n^{T}$ of the plane and $\delta_{d}$ is the difference of distance $d$ of the plane with that of the known plane.

\begin{tabular}{|c|c|c|c|c|c|c|}
\hline Noise & $\bar{\Delta}$ & $\Theta$ & $\sigma_{3} / \sigma_{2}$ & Plane pair $\left(n^{T},-d\right)$ & $\delta_{n}$ & $\overline{\delta_{d}}$ \\
\hline \pm 1.5 & $-0.910^{-7}$ & $-0.2010^{-12}$ & $0.1810^{-3}$ & $\begin{array}{c}\mathbf{( - 0 . 2 2 7 5 2 7}, \mathbf{- 0 . 9 3 4 7 6 1 , 0 . 2 7 2 8 6 1 , ~ 1 . 1 7 5 7 1 )} \\
(-0.954314,0.298090,-0.0206877,8.02997)\end{array}$ & 0.17 & 0.03 \\
\hline \pm 2.5 & $-0.910^{-7}$ & $0.1610^{-11}$ & $0.3010^{-3}$ & $\begin{array}{c}\mathbf{( - 0 . 2 2 8 9 7 2}, \mathbf{- 0 . 9 3 4 9 3 3 , 0 . 2 7 1 0 5 8 , ~ 1 . 1 9 5 8 2 )} \\
(-0.954289,0.298216,-0.0199881,8.02415)\end{array}$ & 0.30 & 0.05 \\
\hline \pm 3.5 & $0.1610^{-6}$ & $0.5010^{-11}$ & $0.5110^{-3}$ & $\begin{array}{c}\mathbf{( - 0 . 2 3 0 8 4 8 ,}-\mathbf{- 0 . 9 3 5 0 1 7}, \mathbf{0 . 2 6 9 1 6 9 , 1 . 2 1 8 4 0 )} \\
(-0.954243,0.298441,-0.0188021,8.01351)\end{array}$ & 0.45 & 0.07 \\
\hline \pm 4.5 & $0.910^{-6}$ & $0.1010^{-10}$ & $0.9210^{-3}$ & $\begin{array}{c}\mathbf{( - 0 . 2 3 3 1 5 4 , - 0 . 9 3 5 0 0 7 , ~ 0 . 2 6 7 2 1 0 , ~ 1 . 2 4 3 2 8 ) ~} \\
(-0.954174,0.298761,-0.0171345,7.99810)\end{array}$ & 0.62 & 0.09 \\
\hline
\end{tabular}

Table 2: Reconstruction results for the second simulated conic in space with different pixel errors for image points before fitting.

perturbation for the discreted conic points, then fit the disturbed points to get the new conic. The closeness of the set of the conics generated in the second image can be deduced from Figure 3 in which four of them are displayed.

Table 3 shows the computed $\Delta$ for each pair of conics. The absolute value of $\Delta$ increases with the increasing discrepancy of conic pairs. Note that as $C_{1}$ is a slightly deformed version of $C_{0}$ and $C_{1}^{\prime}$ of $C_{0}^{\prime}$, it is quite reasonable that $C_{1}$ is as close to $C_{0}^{\prime}$ as to $C_{1}^{\prime}$, as suggested in the table.

\begin{tabular}{|c|c|c|c|c|c|c|c|}
\hline \hline$\Delta$ & $C_{0}^{\prime}$ & $C_{1}^{\prime}$ & $C_{2}^{\prime}$ & $C_{3}^{\prime}$ & $C_{4}^{\prime}$ & $C_{5}^{\prime}$ & $C_{6}^{\prime}$ \\
\hline$C_{0}$ & $\mathbf{0 . 2 0 1 0 ^ { - 1 5 }}$ & $-0.6210^{-5}$ & $-0.8210^{-4}$ & $-0.4710^{-4}$ & $0.2410^{-3}$ & $0.5010^{-2}$ & $0.2610^{-1}$ \\
\hline$C_{1}$ & $\mathbf{- 0 . 4 6 1 0 ^ { - 5 }}$ & $\mathbf{0 . 6 6 1 0 ^ { - 5 }}$ & $-0.4210^{-4}$ & $-0.8110^{-4}$ & $0.1810^{-3}$ & $0.5110^{-2}$ & $0.2610^{-1}$ \\
\hline
\end{tabular}

Table 3: The computed $\Delta$ for each pair of conics $C_{i}$ and $C_{j}^{\prime}, C_{i}$ is a conic of the first image and $C_{j}^{\prime}$ a conic of the second image.

\subsection{The experiments with real images}

\subsubsection{Wooden house images}

We first used a wooden house image sequence that we have been frequently used for self-calibration. Each camera position is calibrated with respect to a coordinate frame associated to the objects in view. Figure 4 illustrates one of the images in which we can see the calibration coordinate frame associated to the scene and the three conics we used for experimentation. The stereo pair of images appear in Figure 5 in which their contour images are displayed. 


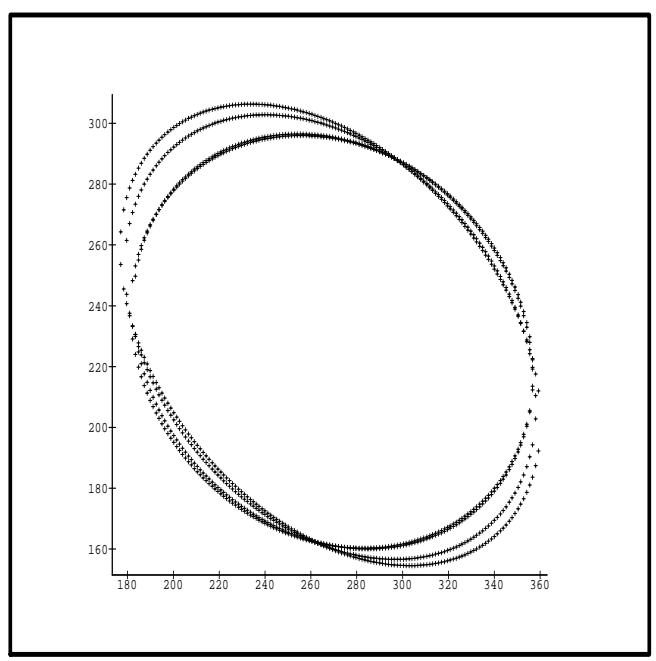

Figure 3: The four conics of the set of generated similar conics. If we look at conics from right to left in the down-left part of the conics, they correspond to $C_{4}, C_{0}, C_{5}$ and $C_{6}$.

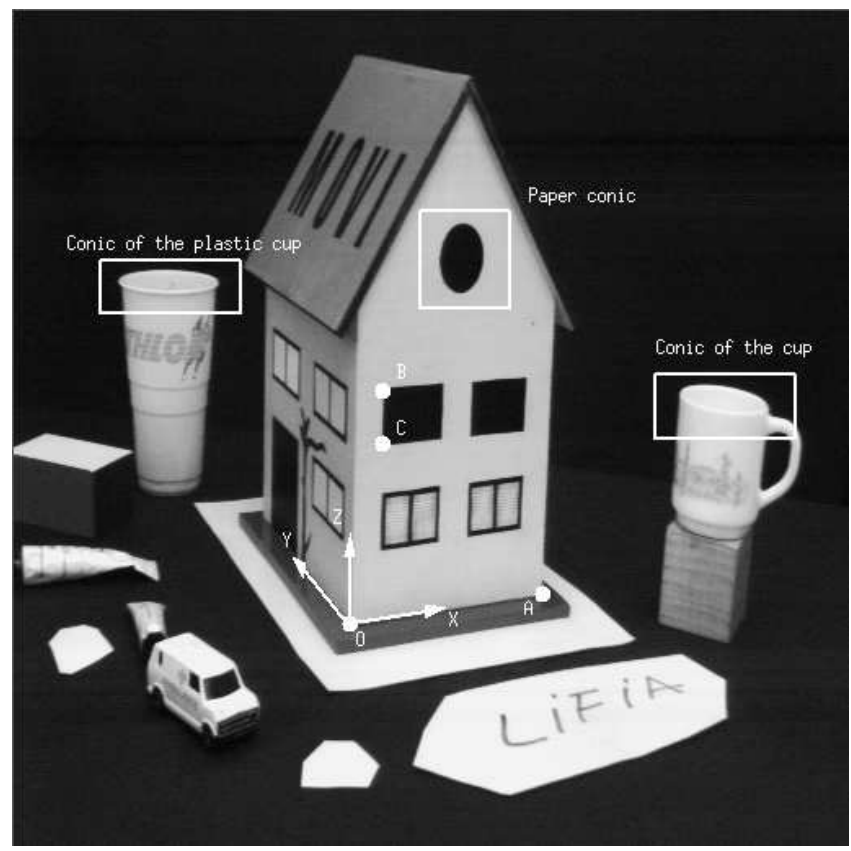

Figure 4: One of the two original images in which the coordinate system for calibration and the conics used in the experimentation are marked. To have an idea of the real size of the object, $O A$ and $B C$ are measured as $13.75 \mathrm{~cm}$ and $2.5 \mathrm{~cm}$.
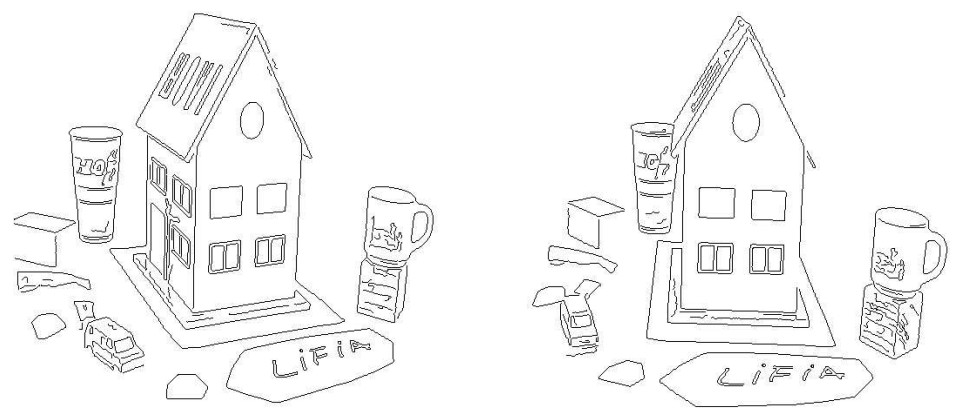

Figure 5: The contour images of the stereo pair of images. 
The pair of images is treated by a Canny-like edge detector, then linked into contour chains. The contour chains of the three conics, the paper conic, the conic of the cup and the conic of the plastic cup are selected by hand from the contour chains. For automatic selection of contour chains of conics, one may refer to [30]. The selected contour chains are then fitted by the procedure described above. Figure 6 illustrates the contour chains and the fitting results of the three conics.

(a).

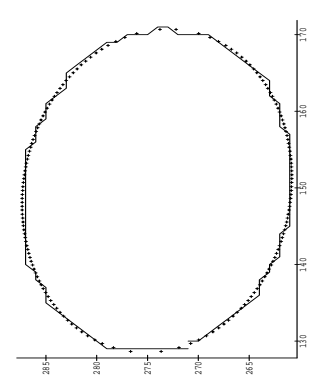

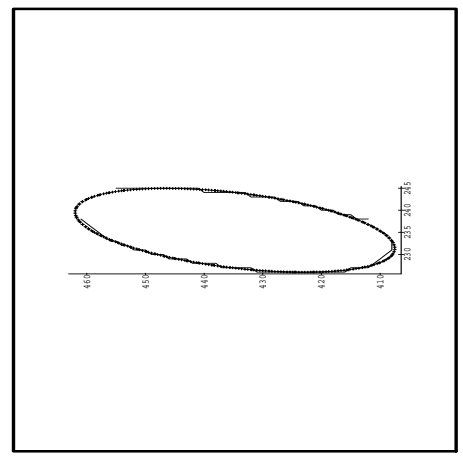

(c).

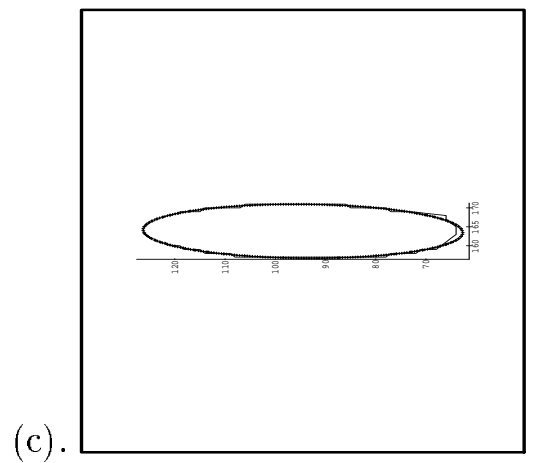

Figure 6: The contour chains to be fitted are displayed in line segments and the fitted conics are dotted. (a): the paper conic. (b): the conic of the cup. (c): the conic of the plastic cup.

The correspondences of conics through two images are unambiguously established by comparing the computed $\Delta$ of Table 4.

\begin{tabular}{|c|c|c|c|}
\hline \hline$\Delta$ & paper & cup & plastic \\
\hline paper & $\mathbf{- 0 . 0 2}$ & 358. & -0.9 \\
\hline cup & 2595. & $\mathbf{- 0 . 1}$ & 1034. \\
\hline plastic & $\mathbf{- 2 8 .}$ & 5214. & $\mathbf{- 0 . 6}$ \\
\hline \hline
\end{tabular}

Table 4: Results of the computation of $\Delta$ in order to establish the correspondences of the conics. The raw entries correspond to the conics of the first image and the column entries to those of the second image.

The reconstruction results are illustrated in Table 5. To have an idea of the reconstruction results, each plane equation is put under the form $\left(n^{T},-d\right)$ where $n^{T}$ is the unit normal direction vector of the plane and $d$ is the distance of the origin to the plane. The plane which corresponds to the real solution, checked by applying the visibility test, is marked in bold font in the plane pair. Recall that the coordinate frame for calibration is illustrated in Figure 4, the paper conic lies in the vertical plane $y=0$; The conics of the cup and the plastic cup lie almost in the planes parallel to the horizontal plane, and the plastic cup is little higher than the cup. All these facts are confirmed by the reconstruction results.

\begin{tabular}{|c|c|c|c|c|}
\hline & $\Delta$ & 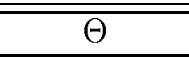 & $\overline{\sigma_{3} / \sigma_{2}}$ & 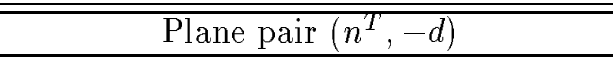 \\
\hline paper & -0.02 & $-0.510^{-9}$ & 0.032 & $\begin{array}{c}\mathbf{- 0 . 0 7 0 7 , ~ 0 . 9 9 3 0 , 0 . 0 9 4 4 5 , - 0 . 4 8 4 5 )} \\
(0.9501,0.3115,-0.01362,-5.855)\end{array}$ \\
\hline cup & -0.1 & $-0.110^{-9}$ & 0.018 & $\begin{array}{c}(\mathbf{- 0 . 0 5 6 4 5 ,}, \mathbf{- 0 . 0 3 2 8 6 , 0 . 9 9 7 9 , - 9 . 4 8 6 )} \\
(0.6811,-0.2470,0.6893,-26.16)\end{array}$ \\
\hline plastic & -0.6 & $-0.1410^{-8}$ & 0.34 & $\begin{array}{c}(\mathbf{0 . 0 2 3 5 2}, \mathbf{- 0 . 0 4 4 4 2 ,} \mathbf{0 . 9 9 8 7}, \mathbf{- 1 0 . 7 8 )} \\
(-0.4283,0.1803,0.8855,-18.22)\end{array}$ \\
\hline
\end{tabular}

Table 5: The reconstruction results of the three conics of the wooden house images. 


\subsubsection{Breakfast images}

We then used a real stereo system coupled to a robot, the stereo system is off-line calibrated with a special calibration objet. The image pair of Figure 7 is taken by this stereo system. The process from edge detection to conic fitting is the same as in the above example. The fitted conics are shown in Figure 8.
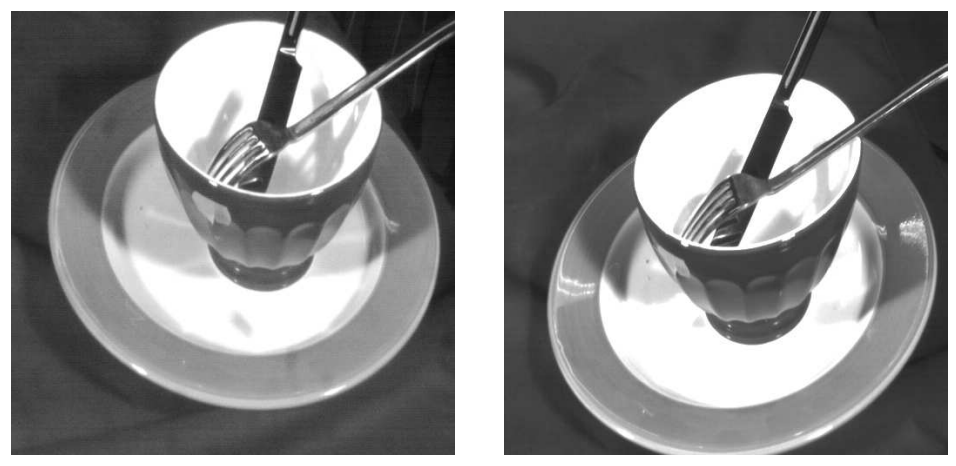

Figure 7: The initial stereo pair of breakfast images.

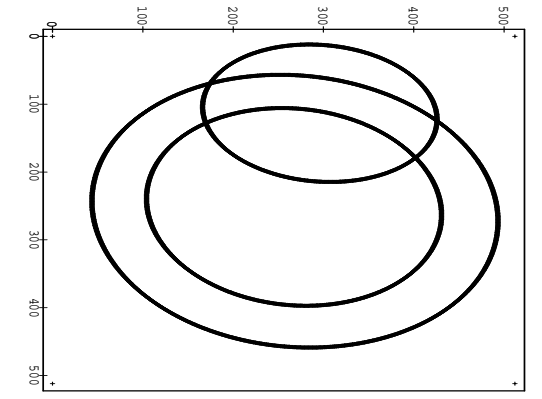

Figure 8: Fitting of three conics in one of the breakfast images.

The correspondences are unambiguously established based on the pairwise $\Delta$, shown in Table 6 .

\begin{tabular}{|c|c|c|c|}
\hline \hline & bowl & dish inside & dish outside \\
\hline bowl & $\mathbf{- 0 . 0 0 1}$ & -15.0 & -3.7 \\
\hline dish inside & -6.0 & $\mathbf{- 0 . 0 0 0 1}$ & 0.54 \\
\hline dish outside & -9.4 & 3.8 & $\mathbf{- 0 . 0 0 0 5}$ \\
\hline \hline
\end{tabular}

Table 6: The computation of $\Delta$ for each pair of conics of two images. The raw entries correspond to the conics of the first image and the column entries to those of the second image.

Table 7 shows the reconstruction results. To have a rough idea of the reconstruction quality, the heights of the conics from the ground, measured with a ruler, are respectively $8.5 \mathrm{~cm}$ for bowl, $3.0 \mathrm{~cm}$ for dish outside and $2.3 \mathrm{~cm}$ dish inside. That makes a difference of $5.5 \mathrm{~cm}$ between bowl and dish outside border and $0.7 \mathrm{~cm}$ between dish inside and outside. Obviously the planes on which conics lie should be all parallel to the ground. The computed difference of the heights are $5 \mathrm{~cm}$ for $5.5 \mathrm{~cm}$ 
and $0.8 \mathrm{~cm}$ for $0.7 \mathrm{~cm}$. The difference of plane orientations are $2.6^{\circ}$ between bowl and inside and $1.7^{\circ}$ between inside and outside border.

Quantitative conclusions can not be drawn from this due to inaccuracy of camera calibration and irregularity of objects. Another important factor is that the porcelain objects have smoothed borders, their image contours have the effect of a moving tangential contour.

\begin{tabular}{|c|c||c|c||c||}
\hline \hline & $\Delta$ & $\Theta$ & $\sigma_{3} / \sigma_{2}$ & Plane pair $\left(n^{T},-d\right)$ \\
\hline bowl & -0.001 & 48.6 & 0.008 & $\begin{array}{c}\mathbf{( 0 . 9 1 8 1 , - 0 . 0 5 0 9 2 , - 0 . 3 9 3 0 , 1 1 . 8 5 )} \\
(-0.1349,-0.9518,0.2753,8.492)\end{array}$ \\
\hline dish inside & -0.0001 & 274.2 & 0.05 & $\begin{array}{c}\mathbf{( 0 . 9 2 1 0 , - 0 . 0 9 3 0 7 , - 0 . 3 7 8 2 , 6 . 0 9 3 )} \\
(-0.1248,-0.9425,0.3100,7.172)\end{array}$ \\
\hline dish outside & -0.0005 & 230.2 & 0.03 & $\begin{array}{c}\mathbf{( 0 . 9 1 3 1 , - 0 . 0 7 8 4 3 , - 0 . 4 0 0 1 , 6 . 8 8 0 )} \\
(-0.1430,-0.9431,0.3002,7.377)\end{array}$ \\
\hline \hline
\end{tabular}

Table 7: The reconstruction results of the three conics of the breakfast images.

Notice that in this experiment the computed $\Theta$ in Table 7 are much higher than in the previous examples. However the ratios of singular values are small enough to indicate the numerical rank of the matrix $C . \Theta$ is related to the scale of the problems.

\subsection{Computation of the invariants}

We have experimented the computation of invariants with the breakfast images.

In this experimentation, the fundamental matrix is first extracted from the two projection matrices provided by the stereo calibration. Then, the two projection matrices up to a collineation are realised by the formule 4 . The conics are then projectively reconstructed. It follows the invariants are computed from this projective reconstruction.

\begin{tabular}{|c|c|c|c|}
\hline \hline & bowl and dish outside & bowl and dish inside & dish inside and dish outside \\
\hline 1 & -17.9643 & 13.6358 & 11.1248 \\
\hline 2 & 2.1267 & 0.6532 & 4.42365 \\
\hline 3 & 2.1136 & 0.676919 & 4.42377 \\
\hline 4 & $\mathbf{4 . 0 1 9 2}$ & $\mathbf{4 . 0 1 4 2 1}$ & $\mathbf{4 . 0 0 0 8 1 6 7}$ \\
\hline \hline
\end{tabular}

Table 8: The results of the computed invariants from the projective reconstruction of the conics.

As we know that three conics are three circles all parallel to the ground, for a pair of parallel circles, the invariant equals 4 according to the formule (3).

As we have the full calibration of the stereo, we have also performed first the Euclidean reconstruction, then compute the invariants from the Euclidean space conics.

\begin{tabular}{|c|c|c|c|}
\hline \hline & bowl and dish outside & bowl and dish inside & dish inside and dish outside \\
\hline 1 & $\mathbf{4 . 0 0 6 5 7 8 9}$ & 0.645893 & 4.42348 \\
\hline 2 & 2.08307 & $\mathbf{4 . 0 1 1 8 1 9}$ & 4.454254 \\
\hline 3 & 2.121461 & 0.3099998 & $\mathbf{4 . 0 5 5 1 1 4}$ \\
\hline 4 & 1.641428 & 0.64579351 & 4.4232326 \\
\hline \hline
\end{tabular}

Table 9: The results of the computed invariants from Euclidean reconstruction of the conics.

The computed invariants are very accurate and stable in both cases. 


\section{Discussion}

This paper proposed an algebraic invariant for a pair of non-coplanar conics in space with the help of projective geometry and classical invariant theory. The relationship between the geometric invariant (in terms of cross ratios) and the algebraic invariant is also established. Algebraic invariants of other configuration composed of conics can be developed in a similar way. Instead of considering the system of a pair of quadratic forms, we can consider the simultaneous invariants of linear forms and quadratic forms.

It is also interesting to consider the special case where one of the conic is the absolute conic [22]. The invariant properties associated to a pair of conics are converted into the metric properties of one conic with respect to the absolute conic.

In order to compute this invariant from two uncalibrated images of the conics, we have proposed a solution to conic reconstruction from two images and conic correspondence between two images within a unified framework for both projective and Euclidean case. We derived two polynomial conditions $\Delta=0$ and $\Theta=0$ to establish the correspondences of conics. It is also shown that the conic reconstruction is generally ambiguous up to two solutions, and only unique for non transparent objects in Euclidean case.

The conic reconstruction method shown is simpler and more stable in comparison with existing methods, as the intrinsic properties of the problem are fully exploited. The experimental results based on both simulated and real images confirm that $\Delta$ is a discriminative correspondence criterion and the reconstruction method is accurate and numerically stable.

\section{Acknowledgements}

This work is partly supported by European Esprit BRA projects Viva which is gratefully acknowledged.

\section{References}

[1] E.B. Barrett, M.H. Brill, N.N. Haag, and P.M. Payton. Invariant linear methods in photogrammetry and model-matching. In J. Mundy and A. Zisserman, editors, Geometric Invariance in Computer Vision, pages 277-292. The MIT press, 1992.

[2] F.L. Bookstein. Fitting conic sections to scattered data. Computer Graphics and Image Processing, 9:56-71, 1979.

[3] S. Carlsson. Projectively invariant decomposition of planar shapes. In J. Mundy and A. Zisserman, editors, Geometric Invariance in Computer Vision, pages 267-276. The MIT press, 1992.

[4] J. Dixmier. Quelques aspects de la théorie des invariants. Gazette des Mathématiciens, 43:39-64, January 1990.

[5] E. B. Elliott. An Introduction to the Algebra of Quantics. Chelsea Publishing Company, New York, second edition, 1913.

[6] O. Faugeras. What can be seen in three dimensions with an uncalibrated stereo rig? In G. Sandini, editor, Proceedings of the 2nd European Conference on Computer Vision, Santa Margherita Ligure, Italy, pages 563-578. Springer-Verlag, May 1992.

[7] O. Faugeras. Three-Dimensional Computer Vision - A Geometric Viewpoint. Artificial intelligence. M.I.T. Press, Cambridge, MA, 1993. 
[8] O.D. Faugeras, Q.T. Luong, and S.J. Maybank. Camera Self-Calibration: Theory and Experiments. In G. Sandini, editor, Proceedings of the 2nd European Conference on Computer Vision, Santa Margherita Ligure, Italy, pages 321-334. Springer-Verlag, May 1992.

[9] M. Ferri, F. Mangili, and G. Viano. Projective pose estimation of linear and quadratic primitives in monocular computer vision. Computer Vision, Graphics and Image Processing, 58(1):66-84, July 1993.

[10] D. Forsyth, J.L. Mundy, A. Zisserman, C. Coelho, A. Heller, and C. Rothwell. Invariant descriptors for 3D object recognition and pose. IEEE Transactions on PAMI, 13(10):971-991, October 1991.

[11] G.H. Golub and C.F. Van Loan. Matrix Computation. The Johns Hopkins University Press, Baltimore, 1989.

[12] P. Gros and L. Quan. Projective Invariants for Vision. Technical Report RT 90 IMAG - 15 LIFIA, Lifia-Irimag, Grenoble, France, December 1992.

[13] P. Gros and L. Quan. 3D projective invariants from two images. In Geometric Methods in Computer Vision II, SPIE's 1993 International Symposium on Optical Instrumentation and Applied Science, pages 75-86, July 1993.

[14] R. Hartley. Invariants of points seen in multiple images. Technical report, G.E. CRD, Schenectady, 1992.

[15] R. Hartley, R. Gupta, and T. Chang. Stereo from uncalibrated cameras. In Proceedings of the Conference on Computer Vision and Pattern Recognition, Urbana-Champaign, Illinois, USA, pages $761-764,1992$.

[16] R. A. Johnson. The conic as a space element. Trans. Am. Math. Soc., 23:335-368, 1914.

[17] K. Kanatani. Geometric Computation for Machine Vision. Oxford Science Publications, Oxford, 1993.

[18] K. Kanatani and W. Liu. 3D interpretation of conics and orthogonality. Computer Vision, Graphics and Image Processing, 58(58):286-301, 1993.

[19] Q.T. Luong. Matrice Fondamentale et Autocalibration en Vision par Ordinateur. Thèse de doctorat, Université de Paris-Sud, Orsay, France, December 1992.

[20] S. Ma. Conics-based stereo, motion estimation, and pose determination. International Journal of Computer Vision, 10(1):7-25, 1993.

[21] S.D. Ma, S.H. Si, and Z.Y. Chen. Quadric curve based stereo. In Proceedings of the 11th International Conference on Pattern Recognition, The Hag, Netherland, pages 1-4, 1992.

[22] S.J. Maybank. The projection of two non-coplanar conics. In J. Mundy and A. Zisserman, editors, Geometric Invariance in Computer Vision, pages 105-119. MIT Press, 1992.

[23] R. Mohr, L. Quan, and F. Veillon. Relative 3D reconstruction using multiple uncalibrated images. The International Journal of Robotics Research, 1995. to appear.

[24] J. Mundy, D. Kapur, S. Maybank, P. Gros, and L. Quan. Geometric interpretation of joint conic invariants. In J. Mundy and A. Zisserman, editors, Geometric Invariance in Computer Vision, pages 77-86. MIT Press, 1992. 
[25] J. Mundy and A. Zisserman. Introduction - towards a new framework for vision. In J. Mundy and A. Zisserman, editors, Geometric Invariance in Computer Vision, pages 1-39. MIT press, 1992.

[26] J.L. Mundy and A. Zisserman, editors. Geometric Invariance in Computer Vision. MIT Press, Cambridge, Massachusetts, USA, 1992.

[27] L. Quan. Polynomial Conditions for Correspondence and Closed Form Solutions for Reconstruction of Conics from a Strongly or Weakly Calibrated Stereo Pair of Images. Technical Report RT 115 IMAG 24 LIFIA, Lifia-ImaG-INRia Rhône-Alpes, 1994.

[28] L. Quan. Invariants of six points and projective reconstruction from three uncalibrated images. IEEE Transactions on PAMI, 17(1), January 1995.

[29] L. Quan, P. Gros, and R. Mohr. Invariants of a pair of conics revisited. Image and Vision Computing, 10(5):319-323, June 1992.

[30] L. Quan and R. Mohr. Using geometric model guided active contour method for traffic sign detection. In Proceedings of IAPR International Workshop on Machine Vision Application, Tokyo, Japan, 1990.

[31] L. Robert and O. Faugeras. Relative 3D positionning and 3D convex hull computation from a weakly calibrated stereo pair. In Proceedings of the 4 th International Conference on Computer Vision, Berlin, Germany, pages 540-544, May 1993.

[32] R. Safaee-Rad, I. Tchoukanov, B. Benhabib, and K.C. Smith. 3D pose estimation from a quadratic curved feature in two perspective views. In Proceedings of the 11th International Conference on Pattern Recognition, The Hag, Netherland, pages 341-344, 1992.

[33] J.G. Semple and G.T. Kneebone. Algebraic Projective Geometry. Oxford Science Publication, 1952.

[34] J.A. Todd. Projective and Analytical Geometry. Sir Isaac Pitman \& sons, Ltd., 1947.

[35] I. Weiss. Projective invariants of shapes. In Proceedings of DARPA Image Understanding Workshop, Cambridge, Massachusetts, USA, pages 1125-1134, 1988.

[36] A. Zisserman, D.A. Forsyth, J.L. Mundy, and C.A. Rothwell. Recognizing general curved objects efficiently. In J. Mundy and A. Zisserman, editors, Geometric Invariance in Computer Vision, pages 228-265. The MIT press, 1992.

[37] A. Zisserman, C. Marinos, D.A. Forsyth, J.L. Mundy, and C.A. Rotwell. Relative motion and pose from invariants. In Proceedings of the British Machine Vision Conference, Oxford, England, pages $7-12$, September 1990 . 